QL

375

G43

INVERT.

ZOOL. 


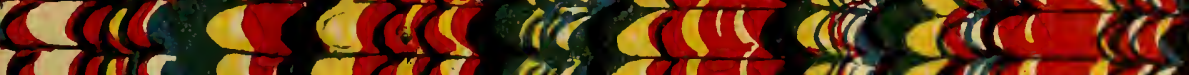

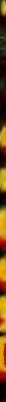

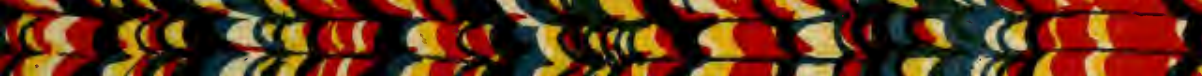
sis cie cer erican ere es is $\operatorname{coser} r a$ ciscra arcis

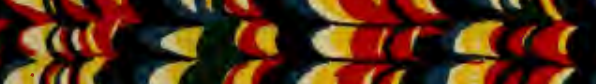

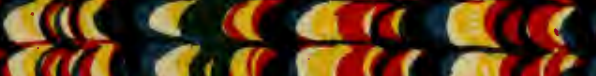
irgerestas ing en cescis (ail $\$ 8$

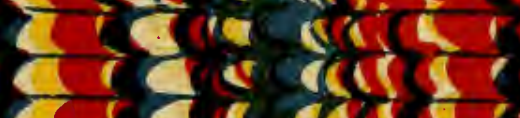
aite 3010

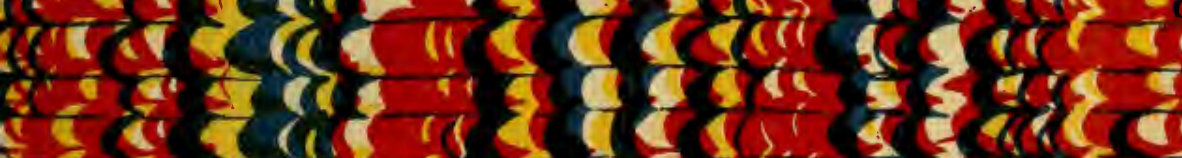
- ertencercese

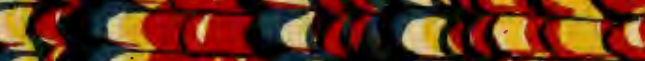
- \& uigereng त) दो

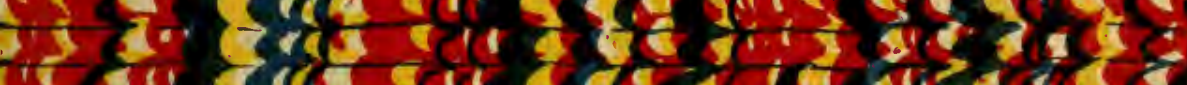

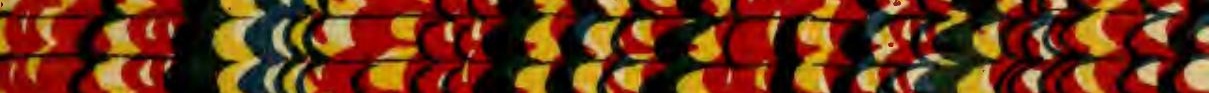

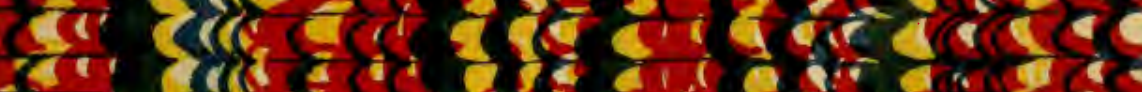

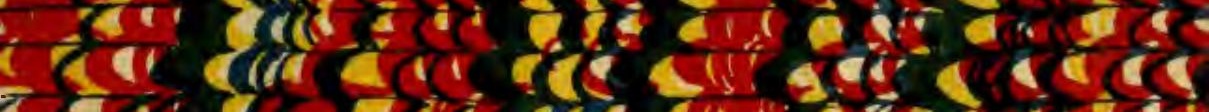

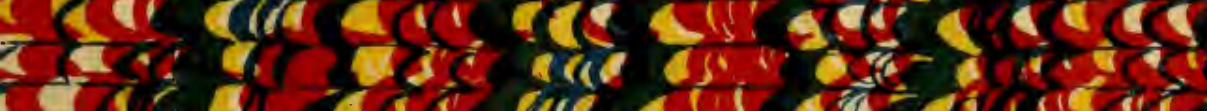
हैe icis at acts 



244750 
,

' 


\section{Ueber Diphyes turgida n. sp., nebst Bemerkungen über Schwimmpolypen,}

von-

\section{Dr. Carl Gegenbaur. *)}

Mit Tafel XXIII.

In meinem in dieser Zeitschrift (Bd. V, Heft 2 u. 3) enthaltenen Aufsatze über die Schwimmpolypen, habe ich noch nachträglich bemerkt, dass ich in Folge einer aus dort angeführten Gründen leicht erklïrlichen Verwechslung eine Diphyes-Art als Diphyes gracilis heschrieben habe, welche schon von Kölliker als Diphyes Sieboldii eingeführt war. Es ergab sich nun, dass das von mir als D. Sieboldii citirte Thier eine neue, bis jetzt unbeschriebene Art sei, welche ich zur Ergänzung meines vorerwähnten Aufsatzes hier näher beschreiben werde. Ich benenne sie

\section{Diphyes turgida.}

Die beiden Schwimmstücke (Fig. $(A B$ ) sind fast gleich lang, und wenn es sich triff, dass das hintere kleiner ist, so rührt dieses daher, dass es früher einmal zu Verluste gegangen und sich späterhin neubildete, ein Umstand, der, wie ich schon früher erwïhnte, bei den Diphyiden gar nicht selten vorkommt.

Das vordere Schwinmstuck $(A)$ besteht aus einem vierseitigen Körper, der nach vorn pyramidal sich zuspitzt. An der Seite treten die Begrenzungsflächen in stumpfen Winkeln zusammen, indess sie vorn und hinten (bei der Lage, in der das Thier in der Abbildung sich finclet, unten und oben) unter mehr scharfen Kanten sich vereinigen. Die hintere (obere) Kante bildet eine ziemlich weit vorspringende Crista, und ist unten gegen die Basis des Schwimmstucks źu schräg abgeschnitten, an welehe Flïche sich das hintere Schwimmstuck anlegt. An der Basis des vordern Schwimmstücks befindet sich die kreisrunde Oeffnung des Schwimmsacks (Fig. $1 A a$ ), die von einer contractilen 
Kreismembran (Velum) umgeben wird. Gleichfalls an der Basis entspringt im rechtem Winkel eine dünne, abgerundete Lamelle $(b)$, die einen, am Anfangstheile des hintern Schwimmstucks befindlichen, zum Durchtritte des Stammes bestimmten Halbkanal theilweise bedeckt, und auf diese Weise ein mögliches Eindringen des Stammes in die Höhle des Schwimmsackes, oder selbst eine Vorlagerung vor die Muindung desselben verhindert. Der Schwimmsack (Fig. $1 A c$ ) bildet eine weite Höhle; die fast ganz genau die äussere Form des Schwimmstucks nachahmt. Nahe an der Mündung des Schwimmsacks findet sich eine fast rings um ihn verlaufende Einbuchtung. Die Gefässvertheilung auf dem Schwimmsacke ist folgende: Nicht weit von der Schwimmsackmündung und nur durch die vorerwähnte halbkreisförmige Lanelle von ihr getrennt, sieht man vom Stamme einen Kanal abgehen, der, am Schwimmsacke angelangt, nahe an der Mündung desselben sich in vier Aeste spaltet; der eine davon liegt in der oberen Medianlinie und läuft gerade auf der Ober-(oder Hinter-) Seite nach rorn zur Spitze, auf welcher er umbiegt, un zur vordern (untern) Seite zu gelangen und schliesslich in einen wie gewöhnlich die Schwimmsackmündung umziebenden Kreiskanal einzumünden. Der zweite Ast ist gleichfalls unsymmetrisch und tritt gleich nach seinem Ursprunge an den naheliegenden Kreiskanal. Die beiden anderen Gefässäste (Fig. $1 A d$ ) verlaufen gleichmässig auf jeder Seitenfläche des Schwimmsacks nach vorn, beugen sich damn nahe an der Spitze um und gehen in sanft Sförmiger Biegung wiederum zum mehr beregten Cirkelkanale.

Das hintere Schwimustück (Fig. $1 B$ ) ]ässt sich, für sich betrachtet, in seiner Form mit einem an beiden Enden schief abgeschnittenen $\mathrm{Cy}_{y}$ linder vergleichen. Sein vorderes Ende ist genau in eine Vertiefung des vordern Scliwimmstücks eingepasst, und nach unten zu (das Schwimmstuck in liegender Stellung gedacht, vergl. die Abbildung) von einem Halbkanale ausgehöhlt, der nach hinten allmählich flach ausläuft. Dieser Fläche entsprechend erstrecht sich gleichwie beim vordern Schwimmstücke eine dünne Lamelle (Fig. $1 b^{\prime}$ ) weit über das Niveau der Schwimmsackmündung hinaus. Der Rand der Lamelle ist abgerundet. Der Schwimmsack $(c)$ ist meist etwas kleiner als jener des vordern Stücks, doch ist er so ziemlich von derselben Gestalt. Die auf ihm stattfindende Gefässvertheilung ist folgende: Etwa am Ende des vordersten Drituheils des Schwimmsacks geht vom Stamme ein kurzer Kanal herüber, der, wie am vordern Schwimmstücke, sich in zwei Aeste rertheilt. Einer derselben läuft zur Spitze und über dieselbe hinweg gerade zur Mündung des Schwimmsacks; ein gleicher kommt längs der hintern oder Unterseite des Schwimmstücks auf kürzerem Wege zu demselben Ziele, während zwei auf den Seitenflächen des Sacks rerlaufende Aeste (d) erst nachdem sie zur Spitze oder dem 
blinden Ende des Sackes emporgestiegen, sich entschieden der Mündung zuwenden, um dort wie die beiden andern in den bekannten Krciskanal einzumünden.

Der Stamm der Gesammtcolonie beginnt, wie dies bei allen Diphyiden der Fall zu sein scheint, an der Vereinigungsstelle zwischen beiden Schwinmstücken $(e)$, jedoch ausnahmsweise nicht mit einem besondern, zuweilen mit grosszelligen Wandungen versehenen Hohlraume, der, wie ich es von Diphyes quadrivalvis und auch von D. gracilis (D. Sieboldii Kölliker ) beschrieben habe, sich in die Substanz des vordern Schwimmstücks einbettet, sondern er entsteht allein aus der Vereinigung jener beiden Kanäle, deren Verlauf und Verästelung auf den Schwimmsäcken vorhin beschrieben wurde. Die Länge des Stammes betrug in seiner grössten Ausdehnung vier Zoll und trug etrva 40 und weniger entwickelte Einzelthiere ( $f$ ) (Gruppen von Organen der Gesammtcolonie). Das Innere des Stammes ist von einem Hohlraume durchzogen, der einerseits mit den vorerwähnten Kanälen communicirt, andererseits sich in alle vom Stamme entspringenden Organe (mit Ausnahme der Deckstücke) fortsetzt. Feine überall gleich vertheilte Gilien unterhalten eine constante Bewegung der im Stamme enthaltenen Flüssigkeit, und wie hierin, so stimmt auch der übrige Bau des Stammes ganz mit dem überein, was von Kölliker von Diphyes Sieboldii und von mir über D. quadrivalvis erwähnt wurde.

Auch "die Einzelthiere», d. i. die aus einem Polypenleib, Geschlechtskapsel und einer Anzahl Fangfäden nebst einer Deckschuppe zusammengesetzten Organgruppen zeigen im Allgemeinen dieselbe Anordnung mit dem schon früher hierüber Bekanntgemachten, weshalb ich dieses füglich übergehen kann. Je nährer dem Anfange des Stammes, desto unentwickelter sind diese Gruppen. Das Erste, was davon sichtbar wird, ist eine Anzahl von Knospen, die sich blinddarmähnlich verlängern, und von demen eine bald die andere an Grösse überholt hat. Diese wird dann zum Polypenleibe, indess die andern sich zu Fangfäden gestalten, erst später entsteht die Geschlechtsknospe, und nach dieser das Deckstück der Gruppe.

Jedes Deckstück (Fig. 2 $c$ ) sitzt an einer queeren Verbreiterung (a) des Stammes (Fig. $2 b$ ) und wird von einer dünnen, mantelartig um den Polypenstamm geschlagenen Lamelle gebildet, die sich nach rorn dem Stamme anliegend zuschärft, indess sie sich nach hinten zur Aufnahme und zum Schutze der vorerwähnten Tlreile trichterförmig erweitert. An der Ansatzstelle ist die aus hyaliner Substanz gebildete Lamelle am dicksten, während sie sich nach ihren Rändern hin allinählich verjünt. Eine Strecke weit greift der eine Seitenrand über den ihm von der andern Seite entgegenkommenden Theil und bildet demnach hier eine doppelte Hiulle. An seinem untern Rande ist jedes 
Deckstück durch zwei stark prominirende Zacken ausgezeichnet, die bei ihrem constanten Verhalten selbst für einzelne, abgerissene Theile einer Diphyes-Colonie specielle Unterschiedsmerkmale abgeben können.

Verlängerungen der Stammeshöhle in die Substanz der Deckschuppe, wie sich solche z. B. bei Praya finden, sind mir hier ebenso wenig vorgekommen als bei den anderen Diphyes-Arten.

Unter jeder Deckschuppe entspringt ein Polypenleib $(d)$, der an seiner Basis sowohl ein Geschlechtsorgan (e) (Samen- oder Eierkapsel) als auch einen Büschel von Fangfäden $(f)$ trägt. Der erste Abschnitt (etwa ein Dritttheil) eines Polypenleibes ist mit einem aus hellen Zellen bestehenden Wulste umgeben, unter welchen der vordere contractile Theil des Polypenleibes sich fortsetzt. Durch diesen Wulst tritt das nur geringer Erweiterung fähige Ende der Leibeshöhle und communicirt mit dem Hohlraume des gemeinschaftlichen Stammes. Der vordere, längere Abschnitt des Polypenleibes bildet die eigentlich verdauende Höhle. Ihre äusseren Wandungen werden durchgehends aus contractilem Gewebe gebildet, welches bald in der Form von quer-oder longitudinalstehenden Spindelzellen, bald als längere Fasern sich zu erkennen gibt, und die äusserst mannichfachen Bewegungen und Formveränderungen dieses Theiles vermittelt. Nach innen folgt dann ein stark entwickeltes Lager von Epithelialzellen, zwischen denen einzelne grosse Drüsenzellen eingebettet sind, in derselben Weise, wie dies früher schon von anderen Diphyiden beschrieben wurde.

An der Basis jedes Polypenleibes sitzt jedesmal ein Geschlechtsorgan, entweder nur als Knospe, oder in entwickeltem Zustande, so dass man vom Anfange des Stammes bis zu dessen Ende sämmtliche Entwicklungsstadien gleichzeitig überschauen kann.

Die Colonien sind hermaphroditisch, indem die Einzelgruppen bald mit einem männlichen, bald mit einem weiblichen Organe versehén sind. Die Aufeinanderfolge der Geschlechter lässt dabei keine Regelmässigkeit erkennen, und es sind häufig mehrere sich folgende Gruppen gleichen Geschlechtes, bis die nächste wieder einen Wechsel im Geschlechte darbietet. Die jüngsten Formen der "Geschlechtsorgane" erscheinen als runde Knospen (Fig. 7), in welche sich der mit dem Cavum des Stammes communicirende Kanal des Basalstückes des Polypenkörpers fortsetzt, der danu bei weiterem Wachsthum der Knospe sowohl eine Centralhöhle derselben als auch vier vom Knospenstiele ausstrahlende seitliche Längskanäle bildet, welch letztere sich sehliesslich vorn durch einen Ringkanal vereinen. Im Parenchym um den CentralhohIraum entstehen die Geschlechtsproducte. Es bildet sich somit auch hier Alles nach dem Medusentypus, doch kommt dieser hier weniger zur Ausbildung als bei Diphyes quadrivalvis und den Prayiden, da die einer Umbrella analoge Hülle der Geschlechtskapsel 
sich nirgends von dem centralen, die Geschlechtsproducte einschliessenden Zapfen abhebt.

Bei vollständiger Reife stellen Samen - und Eikapseln keulenförmige, $0,6-0,7^{\prime \prime \prime}$ lange Organe vor, von denen die ersteren durch ihre Durchsichtigkeit, die letateren durch schön orangerothe Färbung ausgezeichnet sind. Letzteres Colorit rührt nicht von einem besondern Pigmente her, sondern hat seinen Grund in der Masse der Samenfäden selbst, und ist um so schwächer, je weniger diese entwickelt sind. Bei beiden Geschlechtern ist der vordere Theil der Kapseln durch eine Gruppe kleiner, dunkel contourirter Körner, die mit unreifen Nesselzellen gleichen optischen Eindruck machen, ausgezeichnet.

Samen - und Eikapseln sind, wie schon angedeutet ward, in der Anlage einander völlig gleich, und zeigen erst in einem gewissen Stadium eine Verschiedenheit von einander. Das den hohlen Centralzapfen constituirende Parenchym besteht anfänglich aus runden oder sich polygonal abplattenden Zellen, welche, durchsichtig wie die übrigen Gewebe dieser Thiere, erst nach dem Tode ihre Contouren hervortreten lassen. Wird das Organ zu einer Eikapsel, so nehmen diese Zellen einfach an Grösse zu, und zwar um so mehr, je weiter sie nach vorn liegeu, der Kern wächst dabei rasch zu einem runden hellen Bläschen, dem Keimbläschen, welches einen scharf umschriebenen Körper, den Keimfleck, umschliesst (Fig. 6 b). Um den Kern herum sammeln sich einzelne dunkle Molecüle, während die übrige Dottersubstanz völlig klar und durchsichtig bleibt. So wachsen die Eier allmählich bis zu einer Grösse von $0,08-0,1^{\prime \prime \prime}$, und füllen ohne alle Zivischensubstanz, dicht bei einander liegend, die Eikapsel so aus, dass sie bei völliger Reife häufig Ausbuchtungen der Kapsel verursachen (vergl. Fig. 6). Die Anzahl der in einer Kapsel enthaltenen Eier beläuft sich auf 15-20. Sind die meisten Eier völlig reif, so lösen sich die Kapseln vom Stocke ab, bersteu an ihrem Vordertheile, und die vordersten Eier treten nun ins Wasser, wo sie durch einen endosmotischen Vorgang stark aufquellen und dadurch um das Doppelte oder Dreifache grösser werden. Dic in Grunde der Kapsel befindlichen, noch nicht völlig entwickelten Eier entwickeln sich selbst nach abgelöstor Kapsel noch weiter, bis auch an sie die Reihe des Austretens kommt. Es ist mir wahrscheinlich, dass die nachträgliche Entwicklung dieser letzten Eier auf Kosten der Eikapsel selbst geschieht, wenigstens traf ich bei solchen nur noch wenige Eier bergenden Kapseln die Substanz derselben beträchtlich geschrumpft und die ganze Kapsel von geringeren Dimensionen. Die Bildung eines zweiten Generationsorgans, wie es jetzt als «Ersatzorgan" auch von anderen Diphyiden bekannt ist, habe ich auch hier beobachtet, doch nicht mit derselben Constanz, wie sich mir z. B. bei Abyla darbot. 
Im Falle, dass das noch indifferente Generationsorgan sich zur Samenkapsel bildet, so entstehen in den ebenfalls grösser werdenden Parenchymzellen desselben runde, helle Bläschen, die später frei werden und nach völligem Verschwinden der Membran der Mutterzellen dicht bei einander liegen. Diese Bläschen messén 0,003-0,005"'. Ihr Inlaalt trübt sich allmählich und in gleichem Masse nimmt die ganze anfänglich noch zienslich durchsichtige Kapsel eine weissliche Färbung an, die später in mattes Orangeroth übergeht. An den Blïschen wächst dann an einem Pole ein Fortsatz aus, der sich zu einem feinen Faden gestaltet und so die Bildung des Spermatozoiden beschliesst. Die fertigen Samenelemente, aus einem runden Köpfchen mit einem sich scharf absetzenden langen haarförmigen Anhange bestehend, liegen dann regungslos bei einander und harren des Platzens der Kapsel, um dann ins Wasser zu gelangen, wo sie durch lebhafte Schlängelung des Fadenanhanges unter dem Mikroskope jenes bekannte schöne Schauspiel darbieten. - Die Natur jener endogen gebịldeten Bläschen zu bestimmen, wollte mir niemals gelingen, und alle Versuche, sie als Zellen mir anschaulich zu machen, scheiterten immer daran, dass sie unter keinem Verhältnisse ein Kerngebilde nachweisen liessen. Ebenso wenig weiss ich anzugehen, was aus den anfänglich deutlich sichtbaren Kernen der Parenchymzellen wird, und in welcher Beziehung sie zu der Bildung der Bläschen stehen. Möglicherweise zerfallen sie nach der Entstehung der Bläschen wie die Zellmembranen, und ihre Reste helfen dann jene feinkörnige Substanz mit bilden, die man bei Entleerung einer reifen Samenkapsel niemals zwischen den Spermatozoiden vermisst.

Die Fangfäden (Fig. 4) sitzen gleichfalls an der Basis jedes Polypenkörpers, und zwar zumeist gegenüber den Geschleclitsorganen, wo sie ein dichtes, immer eine ganze Entwicklungsreihe darstellendes Bündel bilden. Wie die Fäden der ỉbrigen Diphyiden, sind auch diese im ausgebildeten Zustande mit secundären Fädchen (Fig. $4 b$ ) besetzt, deren jedes mit einem ovalen Nesselknopfe (c) endet. Ein solches Nesselorgan misst $0,1^{\prime \prime \prime}$ Länge und besteht aus einer, aus mehrfachen Reihen queerliegender stäbchenförmiger Nesselzellen zusammengesetzten Batterie $(d)$, welche meist nach aussen eine schwache Wölbung zeigt. Der obere Theil dieser Batterie besitzt eine gelbbräunliche Färbung, die von diffusem in die Bindesubstanz zwischen die Nesselzellen eingelagertem Pigmente herrührt. An der concaven Seite dieser Batterie finden sich dann noch $6-8$ ausnehmend grosse Nesselzellen $(e)$ von derselben Form, deren Längendurchmesser senkrecht auf dem der vorigen steht. Das ganze linöpfchen wird von einem Pflasterepithel überzogen. Am untern Ende, etwas seitlich und dem Anheftungspunkte des secundären Fadens genähert, entspringt von jedem Nesselknopfe noch ein $0,1 \quad 0,15^{\prime \prime \prime}$ langes Fädchen $(f)$, welches äusserst contractil 
erscheint und im zusammengezogenen Zustande meist eine aus wentgen Windungen bestehende Spirale vorstellt. Die Oberfläche dieses Fädchens ist dicht mit runden Nesselzellen besetzt, deren Faden bald als kurze Spitze, bald als längere starre Borste hervorragt. Der letztere Zustand ist in der Abbildung Fig. 4 wiedergegeben. Wie diese Nesselzellen, so strecken auch jene der Batterie im Knöpfchen oft sämmtlich ihren viel längeren, aber nicht minder "starren Faden hervor, und lassen auf diese Weise in den unscheinbaren Knöpfchen eine furchtbare Waffe erkennen. Immer ist nur Ein Fangfaden vollständig entwickelt vorhanden, der dann $6-10$, in regelmässigen Abständen sitzende, secundäre Fädchen trägt, gleichzeitig sind aber noch $1-2$ Fäden ibrer Ausbildung nahe und können so den ersten im Falle seines Verlustes alsbald ersetzen. Das ganze System der Fangfäden wird von einem Kanale durchzogen, der mit dem Hohlraume des Stammes communicirt und dessen Verzweigungen in den contractilen Terminalfädchen der Nesselknöpfe geschlossen endigen.

Schliesslich will ich noch beifügen, dass es die eben genauer beschriebene Diphyes turgida war, an deren Eiern ich die Entwicklungsgeschichte der Siphonophoren am weitesten verfolgen konnte und verweise in dieser Hinsicht auf meine frühere Abhandlung.

Die besondere Aufmerksamkeit, welche in neuester Zeit von Seite verschiedener Forscher den Schwimmpolypen zu Theil wurde, brachte es mit sich, dass, so wie einerseits die Kenntniss von diesen interessanten Geschöpfen dadurch um ein Beträchtliches gefördert ward, auch andrerseits die Nomenclatur durch Synonyme bedeutend vermehrt, ja man darf sagen, belastet wurde, weshalb es für einen mit diesen Thierformen weniger durch eigene Anschauung Vertrauten nicht gerade leicht ist, sich ohne Weiteres hierin zurecht zu finden und Identisches von Nichtidentischem zu scheiden, wenn diess auch durch die den neueren Arbeiten beigegebenen Abbildungen ermöglicht scheint.

Indem ich nun eine kurze Zusammenstellung der neuerlich bekannt gewordenen Siphonophoren-Arten versuche, beschränke ich mich dabei nur auf jene, welche auch von mir beschrieben wurden, und wovon eine kurze Skizze bereits im ersten Hefte, und eine ausführlichere Darstellung im 2.-3. Hefte des V. Bandes dieser Zeitschrift enthalten ist. Auch jene Verbältnisse sollen in der Kürze beriihrt werden, wo sich in unseren Beobachtungen bemerkenswerthe Differenzen zeigen, oder wo verschiedene Folgerungen davon abgeleitet wurden.

$$
\text { Diphyes gracilis mihi }
$$

wurde schon früher als synonym mit der von Kölliker als D. Sieboldii beschriebenen Art dargestellt, und wenn sich auch zwischen den beider- 
seitigen Beschreibungen einzelne Differenzen ergaben, z. B. uber die Form der Deckstücke, die von Kölliker als nach einer Seite hin offene Schuppen, von mir als trichterförmig zusammengerollte Blättchen dargestellt wurden, so fällt unsere Beschreibung in der Hauptsache zusammen, und der Speciesname D. gracilis muss fernerhin in D. Sicboldii aufgehen. Die von Leuckart ${ }^{1}$ ) im Golfe von Nizza beobachtele Diphyes acuminata stimmt zwar in den arehitektonischen Beziehungen ihrer Schwimmstücke auffallend mit D. Sieboldii uberein, aber der Verlauf der Gefässe am hintern Schwimmstücke und der an demselben sich findende geschlossene Kanal zum Durchtritte des Stammes, so wie die Unisexualität der Colonien lässt sie als eine verschiedene Species auffassen.

\section{Diphyes quadrivalvis mihi.}

C. Vogt ${ }^{2}$ ) hatte diese Diphyes früher als Epibulia aurantica bezeichnet, und in seinem neuern Werke $^{3}$ ) erkenne ich sie augenblicklich wieder unter dem Namen Galeolaria aurantiaca., Ich bezog die von mir untersuchten Thiere auf die von Blainville aufgestellte Gattung Sulcoleolaria, und zwar auf die Art quadrivalvis, welche mil noch zwei anderen im Golf von Nizza vorkommen soll, und die nach der in Lesson's hist. nat. des Acalephes (p. 143) aufgeführten Beschreibung jedenfalls eine sehr nahe stehende Form charakterisirt. Da ich jedoch weder Blainville's Atlas, noch jene von Quoy und Gaimard hierüber zu Rathe ziehen konnte, so muss ich die Frage uber die Identität von Galeolaria und Sulcoleolaria noch unentschieden lassen. Eine Frage erlaube ich mir aber hier aufzuwerfen, nämlich in wiefern es gerechtfertigt sei, Galeolaria als ein von Diphyes verschiedenes Genus beizubehalten? Der Gattungscharakter von Diphyes liegt hauptsächlich in der Form der beiden Schwimmstücke und in der Art ihrer Vereinigung, so wie ferner in der Form der Deckstucke und den Gruppirungsverhältnissen der übrigen Organe an dem gemeinsamen Stamme. In all' diesen Stücken stimmt D. quadrivalvis frappant mit den übrigen Diphyes überein, so dass es nothwendig wäre zur Aufrechthaltung der Gattung Galeolaria zu geringfügigeren Merkmalen seine Zuflucht zu nehmen. Diese finden sich denn auch in den klappenartigen Fortsätzen an der Mündung jedes Schwimmstücks und in der höhern Entwicklung der Geschlechtsorgane. Was den ersten Punkt betrifft, so sind zackenartige Verlängerungen und lamellenförmige Fortsätze an der Mündung der Schwimmstücke bald mehr, bald we-

1) Zoologische Untersuchungen, 4. Heft. Giessen 1833.

2) Zeitschrift für wissenschaftliche Zoologie. Bd. II, 1). 522.

$\left.{ }^{3}\right)$ Recherches sur les Animaux inférieurs de la méditerranée prem. Ilém. 1). 110. 
niger entwickelt bei den meisten bekannteren Diphyes-Arten, ja sogar bei allen, die in der neuesten Zeit genauer untersucht wurden, vorhanden, und wollte man auf diese Verhältnisse Gattungsunterschiede begründen, so würde gar bald das ganze Genus Diphyes in ebenso viele Genera zersplittert als Arten davon bekannt sind. Die höher entwickelte Form der Geschlechtsorgane, die den Medusentypus ausgeprägter zeigen als bei den übrigen Diphyes-Arten, ist deshalb unzureichend, weil der Unterschied nur ein gradueller bleibt und auf den Typus der Gesammtcolonie wenig oder gar nicht influenzirt. Liefern uns doch die nächsten Verwandten der Siphonophoren, nämlich Hydraspolypen, zahlreiche Beispiele, wie sehr die sogenannten Geschlechtsorgane verschieden entwickelt sein können, wie die eine Art derselben Gattung beständig Medusen aufnimmt, während die andere bei ihrer Fortpflanzung nur mit der Production einfacher, Samen oder Eier erzeugender Knospen sich begnügt, ohne dass wir darin einen Grund fänden, hierauf neue Genera zu begründen!

\section{Praya maxima mihi}

wurde auch von Leuckart beobachtet und von demselben unter dem Namen Pr. cymbiformis Delle Chiaje aufgeführt. Die untersuchten Thierstöcke dürften jedoch schwerlich vollständige gewesen sein, da Leuckart sowohl die geschlechtlichen Verhältnisse dieser Thiere verkannt hat, als auch den Schwimmapparat unvollständig beschreibt. Auch Vogt scheint diese-Praya, oder wenigstens ein einzelnes Schwimmstück davon, beobachtet zu haben, wie ich aus einer auf Taf. 16, Fig. 3 gegebenen Abbildung ersehe; es wird aber dort von ihm zu Praya diphyes Blainv. gerechnet, einer Art, die durch die Antrittsstelle der Gefässe zu den Schwimmsäcken der Schwimmstücke und die Verästlung auf denselben scharf von der vorerwähnten verschieden ist.

Meine Beobachtungen über "einfache Diphyiden», namentlich über die von Abyla pentagona stammende Eudoxienform, fanden durch Leuckart's an demselben Thiere angestellte Untersuchungen ihre Bestätigung, so wie auch Vogt für Abyla trigona zu gleichen Resultaten gelangt war, so dass nummehr durch drei unabhäng von einander angestellte und fast gfeichzeitig veröffentlichte Untersuchungsreihen, das Verbalten der "eiufachen Diphyiden» zu den Diphyescolonien definitiv festgestellt scheint. Desscnungeachtet glaube ich hier auf einige hieher bezüglichen Angaben Leuckart's, insofern sie von meinen Beobachtungen abweichen, etwas näher eingeben zu müssen.

Das Eine davon ist nämlich die Art der Befestigung der AbylaAbkömmlinge (Eudoxia cuboides Leuck.) am gemeinschaftlichen Stamme, ein zwar scheinbar unwesentlicher Unstand, aber doch, wie ich glaube, für die Auffassung der Eudoxien, und besonders für die von Leucliart 
versuchte Ausdehnung der Eudoxienproduction auf mehrere DiphyesArten von nicht unbedeutender Wichtigkeit. Leuckart gibt an, dass das kubische Deckstück der Einzelgruppen anfänglich eine sattelförmige Umhüllung um den Stamm bilde, die bei weiterem Wachsthum denselben umschliesse, so dass bei ausgebildetem Deckstücke der Stamm durch den kubischen Körper hindurchtrete; bei der Abtrennung der Eudoxie zerreisse nun der Körperstamm etwa in der Mitte zwischen zwei Anhangsgruppen (Eudoxien) und eine Zeitlang trage die junge Eudoxie noch den Stumpf dieses Stammes, der aus der obern Fläche des Würfels hervorrage, mit sich umber. Wenn dann das obere Ende des kubischen Deckstücks allmählich wachse, dann werde dieser Stumpf auch allmählich verloren gehen. Meine Beobachtungen zeigen von'diesen Leuckart'schen eine beträchtliche Divergenz.

Erstlich sah ich das Deckstück der Einzelgruppen niemals um die ganze Peripherie des Stammes sich bilden, sondern gleichwie die Deckstiucke aller übrigen Siphonophoren, nur nach einer Seite hin, da, wo es zuerst am Stamme in Form einer Knospe zum Vorschein kan. Die obere Fläche des Würfels war daher immer am entferntesten vom Stamme, und nur an seiner vertieften Basalfläche, da, wo Geschlechtsknospe, Magenstück und Fangfäden ihren Ursprung nehmen, stand es mit dem Stamme in Verbindung. Der Längendurchmesser der fertigen Eudoxie fällt daher nicht mil jenem des Stammes zusammen, wie es aus Leuckart's Beschreibung hervorgeht, und auch durch eine Abbildung von ihm dargestellt wird (1. c. Taf. III, Fig. 1), sondern er steht quer zu demselben, bald unter rechtem, bald unter mehr spitzem. Winkel je nach den zufälligen Bewegungen der Colonie. Nach diesen an lebenden Abylacolonien oft wiederholten Untersuchungen musste mir der von Leuckart mitgetheilte Befund um so auffallender erscheinen, und mich veranlassen, die von mir gesammelten Abylen zu einer Revision des Verhaltens der eudoxienförmigen Einzelgruppen zu benutzen. An wohlerhaltenen war zwar immer der ganze Stamm in den bekannten Kanal des grössten Schwimmstücks eingezogen, aber es fanden sich bei einigen nach einer Reihe von weniger entwickelten Sprösslingen noch 2-3 fast vollständig entwickelte Eudoxien, welche sich sämmtlich in queerer Richtung am Stamme angereiht fanden, so dass die obere Fläche des Deckstücks als frei, vom Stamme abgewendet zu erkennen war, was nicht hätte der Fall sein können, wenn der Stamm mitten durch sie sich inserirte. Durch die Einwirkung der conservirenden Flüssigkeit auf sämmtliche contractile Theile der Colonie, welche dadurch völlig undurchsichtig geworden waren, trat das Verhalten der vollkommen pellucid gebliebenen Deckstucke um so deutlicher hervor, und wenn der Stamm sich durch sie hindurchsetzle, so könnte dies schwerlich bei dicser Behandlungsweise sich der Beobachtung entziehen. 
Als einen andern Controverspunkt betrachte ich die von Leuckart aufgestellte Hypothese von der Abstammung seiner Eudoxia campanula. Leuckart kommt durch eine Reihe von Vergleichungen der Deckstücke seiner Diphyes acuminata mit dem Deckstücke der eben bezeichneten Eudoxia zu dem Schlusse, dass erstere sich durch besondere Veränderungen allmählich in die Deckstücke der E. campanula umwandelten, so dass diese somit als abgelöste Einzelgruppen einer Diphyes, und zwar der D. acuminata, zu betrachten seien. Leuckart geht hiebei wohl hauptsäehlich ron der Annahme aus, dass die Bildung des Eudoxiendeckstücks in derselben Weise vor sich gehe, wie dies von ihm für Eudoxia euboides angegeben wurde, dass es sich nämlieh rings um den Stamm entwickle, und dass der Stamm es in seiner Längsachse durehsetze. Da mir bis jetzt ausser Abyla keine Diphyide bekannt ist, welche Eudoxien hervorsprosst, so kann ich natürlicherweise über die Entwicklung der betreffenden Deckstücke anderer Eudoxien keine Angaben machen, und es bleibt nur der Schluss von den Eudoxien der Abyla auf jene mit pyramidalem Deckstücke; die ex analogia genommene Folgerung ist, dass auch die letzteren einen lateralen Ursprung haben, und dass nur an der Basalfläehe, da, wo die übrigen Theile der Eudoxia entspringen, die Auheftung an den gemeinsamen Stamm statt findet. Aber auch von anderer Seite her lassen sich Gründe gegen die in Frage stehende Umwandlung der Diphyes-Deckstücke in solche von Eudoxien anführen. Betrachten wir nämlich die Entstehung der Deckstücke bei verschiedenen Diphyes-Arten (ich habe hier vorzüglich die von mir untersuchten drei Arten in Auge), so tritt uns überall derselbe Typus entgegen, überall entsteht am Stamme eine cinfache Knospe, die sich wulstartig verbreitert und dann sowohl nach oben und unten, als auch seitlich auswächst, ohne jedoch auf letzterem Wege sich zu vereinen, und einen den Stamm vollständig umschliessenden Ring bilden. Während nun der nach oben hin auswachsende Theil des Deekstücks gegen den Stamm zu convergirt, divergirt der raseher wachsende untere Theil, und so entsteht eine trichterförmig um den Stamm gewickelte Lamelle, deren Seitenränder eine Strecke weit über einander greifen. Es bleibt dabei immer am Stamme in gleicher Höhe mit dem Ansatzpunkte des Deekstücks noch ein Theil frei, der nur von den Rändern der Schuppe überragt wird, ohne mit ihnen jedoch in direeter Verbindung zu stehen, so dass dadurch ganz andere Formverhältnisse erseheinen, als wir sie bei den soliden Deckstücken der Eudoxien uns entgegentreten sehen. Obgleich ich nun nicht im Stande bin, die Möglichkeit der Leucliart'schen Hypothesen über die $\Lambda$ bstammung der Eudoxien von Diphyescolonien zu bestreiten oder gar zu widerlegen, so glaube ich doeh deren Unwahrseheinlichkeit hinreichend dargethan zu haben, so wie ich mich zur Annahme berechtigt halte, dass die Bildung des Eudoxiendeckstücks auf dieselbe Weise vor 
sich geht, wie bei den Eudoxien der Abyla. Dass die Einzelgruppen der Diphyescolonien vermöge der Architektonik ihrer Deckschuppen und der Relation ihrer einzelnen Organtlıeile nicht befähigt sind, nach erfolgter Ablösung vom Stamme ein selbständiges Leben fortzuführen, habe ich schon früher nachzuweisen versucht.

Schliesslich füge ich eine systenatische Skizze der Diphyiden bei, worin ich ausschliesslich die in neuerer Zeit bekannt gewordenen Arten aufnahm, da eine bezügliche Verwerthung des ältern Materials aus leicht begreiflichen Gründen eher Vervirrung verursachen möchte.

\section{D i p b y i d a e.}

Zwei Schrvimmstücke am Anfange des Stammes. Die einzelnen Organe sind am Stamme in bestimmter Anzahl auf Gruppen vertheilt. Keine Schwimmblase.

\section{Genus. Pra y a .}

Schwimmstücke neben einander, fast gleich gross, vorn abgerundet. Schwimmsack relativ klein. Deckstücke der Einzelgruppen helmförınig, solide, von mehreren Kanälen durchzogen.

\section{Arten: Pr. diphyes Blainville.}

Schwimmstücke nur lose mit einander verbunden, in jedem Schwimmstücke ein bläschenförmig endender Kanal, der ınit dem Stamme in Verbindung steht. Gefässverlauf auf den Schwimmsäcken gerade, vom hintern Ende derselben bis zum Ringkanal an der Mündung.

\section{Pr. maxima mihi.}

Schwimmstücke ungleich gross. Das kleinere wird vom grössern theilweise umfasst. Nur im kleinern Schwimmstücke ein blind endender Fortsatz des Stammes. Die beiden seitlichen Gefässe verlaufen auf den Schwimmsäcken in vielfachen Biegungen.

\section{Genus. D i ph y es.}

Schwimmstücke hinter einander, das vordere pyramidal zugespitzt. Das hintere in das vordere eingefalzt. Schwimmsäcke lang, kegelförmig. Deckstücke sind dünne, trichterförmig um den Stamm gerollte Lamellen ohne Gefässe.

\section{Arten: D. Sieboldii Kölliker.}

Vorderes Schwimmstück stark zugespitzt, mit einem vorragenden Fortsatze zur Aufnahme des hintern Schwimmstücks. An dessen Mündung zwei starke, divergirende Zacken.

D. quadrivalvis mihi (Galeolaria aurantiaca Vogt).

An der Mündung des vordern Schwiınmstücks zwei klappenförmige Fortsätze, an jener der hintern vier, wovon zwei ausgeschnilten sind. 


\section{D. a cuminata Leuckart.}

In Form der erstern ähnlich. Im hintern Schwimmsticke ein mit Oeffnungen versehener Kanal.

\section{D. turgida mihi.}

An der Mündung des vordern, so wie an jener des hintern Schwimmstücks eine vorstehende abgerundete Lamelle.

\section{Genus. A b y la.}

Schwimmstücke ungleich entwickelt. Das vordere sehr klein, das hintere bedeutend grösser. Deckstücke der Einzelgruppen kubisch oder vielfach ausgezackt. Die einzelnen Gruppen lösen sich vom Stamme und fübren ein selbständiges Leben (Eudoxien).

Arten: Abyla pentagona Eschsch. (Calpe pentagona Q. u. G.).

Hinteres Schwimmstück an jeder Mündung mil fünf vorspringenden Zacken, denen ebenso viel Kanten entsprechen.

Ab. trigona $Q$. und $G$.

An der Mündung des hintern Schwimmstücks nur drei Zacken.

\section{Erkräirung der Abbildungen.}

Fig. 1. Diphyes turgida. $3 \frac{1}{2}$ mal vergrössert, mit ausgedehntem Stamme. $A$ vorderes, $B$ hinteres Schwimmstiick; $a$ a Mündung der Schwimmstuicke; $b$ lamellenartiger Fortsatz an der Mündung des vordern, $b$ an der Mündung des hintern Schwimmstücks; $c$ Schwimmsack; $d$ Gefässe auf demselben; $e$ Anfangstheil des Stammes; $f$ Einzelthiere (einzelne Organgruppen). Männliche Kapseln sind von den weiblichen durch orangerothe Färbung ausgezeichnet.

Fig. 2. Ein Abschnitt des Stammes mit zwei Einzelgruppen; bei stärkerer Vergrösserung; bej der untern Gruppe ist das Deckstück weggelassen; bej beiden der ausgebildete Fangfaden. $a$ der Stamm; $a^{\prime}$ Höhle desselben; $b$ Erweiterung des Stammes an der Ansatzstelle eines Deckstiucks; $c$ Deckstuick; $d$ Polypenleib; $e$ Geschlechtskapsel; $f$ ein Büschel junger Fangfäden, wie $e$ von der Basis des Polypenleibes entspringend.

Fig. 3. Hinteres Schwinmstiick von der Rückseite. a Mündung des Schwimmsacks; $b$ vorstehende Lamelle an derselben; $c$ Schwimmsack; $d$ Antrittsstelle des Kanals an denselben; $e$ e e e die vier Gefässe, in welche sich der Kanal verzweigt.

Fig. 4. Stiick eines Fangfadens, stärker vergrössert. $a$ Hauptfaden; $b$ ein secundärer Fangfaden; $c$ Nesselknopf desselben; $d$ mehrere Reihen von Nesselzellen; $e$ grössere Nesselzellen; $f$ Terminalfaden mit ausgestreckten Fäden seiner Nesselzellen.

Fig. ¿̋. Eine Samenkapsel, auf dem Durchschnitte gesehen. a äussere Hiille (Analogon der Umbrella der Medusen); $b$ Parenchym; $c$ Stiel; $d$ Kanal in letzterem, der mit dem Hohlraum des Stammes in Verbindung steht, und bei seinem Antritt an die Kapsel sich in $e$ den Centralkanal und $f$ oberflachliche in der Hülle verlaufende Gefässe vertheilt; $g$ Einmiindıng der Längskanäle in den Kreiskanal.

Fig. 6. Eicrkapsel, von der Oberfläche gesehen. $a, c, d, f$, wie in Fig. 5; $b$ Eicr; $b^{\prime}$ Keimbläschen derselben; $b^{\prime \prime}$ Keimfleck.

rig. 7. 8. Entwicklungsstadien der Geschlechtskapseln. 

4 


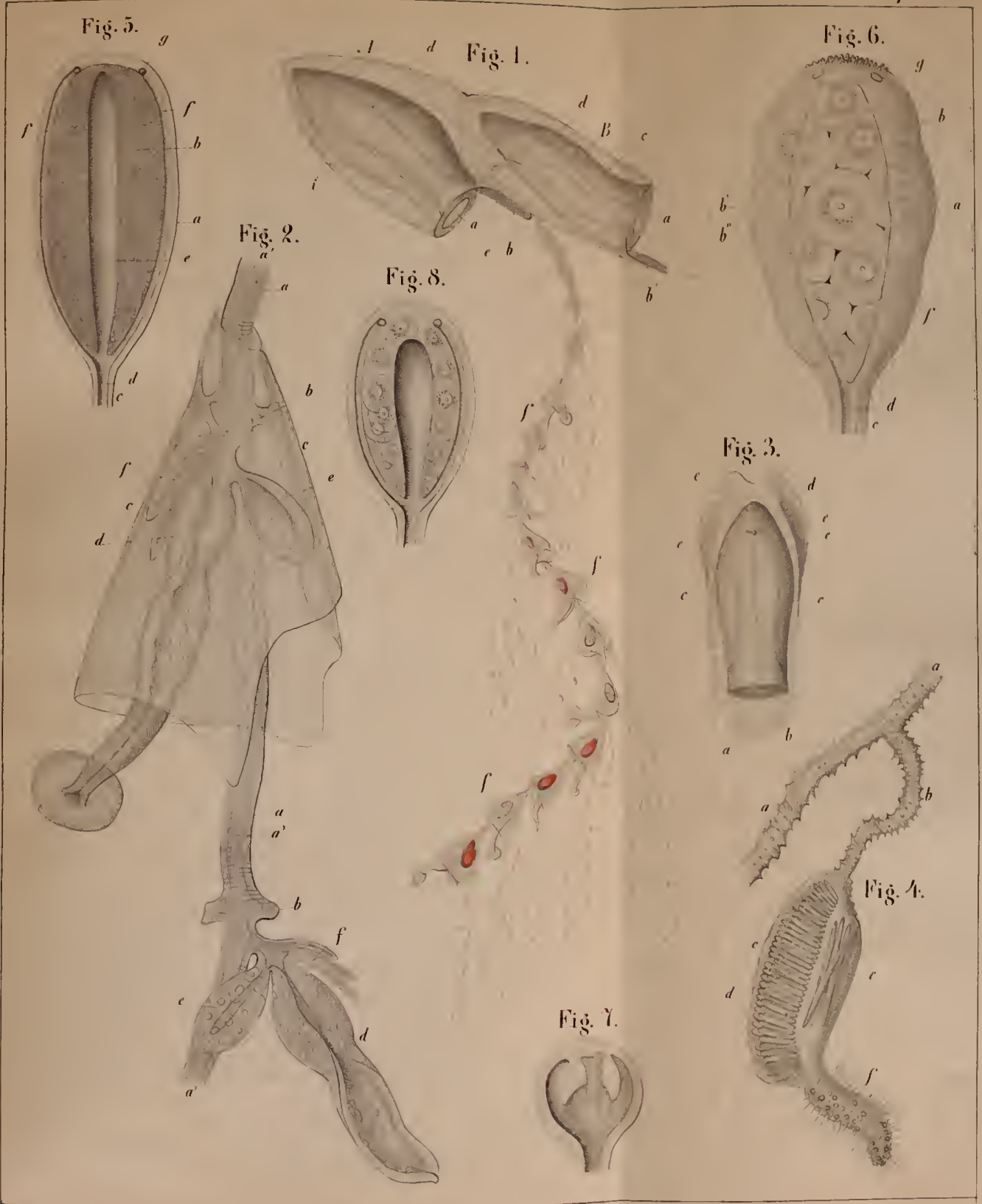







$$
\begin{aligned}
& m q 541765 \\
& \text { ||||||||||||||||||||||!| }
\end{aligned}
$$

